\section{Clinical course, severity and mortality in a cohort of patients with COVID-19 with rheumatic diseases}

The recent outbreak caused by a novel severe acute respiratory syndrome coronavirus 2 disease 2019 (COVID-19) has spread rapidly worldwide, and it has been declared a pandemic by the WHO. ${ }^{1}$ Elder people, male sex and some underlying comorbidities seem to be risk factors for morbidity and mortality, although an immunosuppressive status could favour the infection and the development of complications. ${ }^{2}$ However, as progress is made in the knowledge of the physiopathology of COVID-19, it has been observed that severe respiratory forms occur as a result of an hyperinflammatory status and an excessive production of cytokines. $^{3}$

In this descriptive retrospective study, we aimed to characterise features related to severity and mortality in these patients and the influence of immune modulating drugs on the course of the infection. Patients were included from 25 February 2020 to 8 June 2020 with COVID-19 infection and rheumatic inflammatory diseases from Rheumatology Department of La Paz University Hospital.

One hundred and twenty-two patients were included. One hundred $(82.0 \%)$ were confirmed through nasopharyngeal swabs. Twenty-two patients (18.0\%) exhibited compatible symptoms with compatible lung imaging and/or positive serology. Patients characteristics are shown in table 1.

Variables associated with hospital admission in univariate analysis (table 2) were age (5-year intervals; OR 1.34, 95\% CI 1.17-1.55), prednisone dose >5 mg/day (OR 2.55, 95\% CI 1.07-5.59), chronic pulmonary disease (OR 5.34, 95\% CI 1.47-19.35) and hypertension (OR 4.06, 95\% CI 1.79-9.19). Independent risk factors for hospital admission were methotrexate (OR 2.06, 95\% CI 1.01-5.29) and age (5-year intervals; OR 1.31, 95\% CI 1.11-1.48). No association was found with hydroxychloroquine, other conventional disease-modifying antirheumatic drugs (cDMARDs), targeted synthetic disease-modifying antirheumatic drugs or biological disease-modifying antirheumatic drugs (bDMARDs) or laboratory parameters. Methotrexate treatment was not associated with age, sex, glucocorticoids or subtype of rheumatic disease.

Fourteen patients died $(11.5 \%)$ due to respiratory failure. Nine patients were on cDMARDs (either in monotherapy or in combination), one was on bDMARD (rituximab) and four were taking only oral glucocorticoids. Hydroxychloroquine did not show differences in mortality. On univariate analysis, factors associated with mortality were age (OR 1.60, 95\% CI 1.202.01), arterial hypertension (OR 12.17, 95\% CI 2.58-57.38),

\begin{tabular}{|c|c|}
\hline \multicolumn{2}{|l|}{ Demographics } \\
\hline Female sex, n (\%) & $80(65.6)$ \\
\hline Caucasian ethnicity, n (\%) & $98(80.3)$ \\
\hline Age $($ mean $\pm S D)$ & $58.3 \pm 16.3$ \\
\hline \multicolumn{2}{|l|}{ Comorbidity, n (\%) } \\
\hline Hypertension & $48(39.3)$ \\
\hline Obesity & $27(23.6)$ \\
\hline Chronic pulmonary disease & $20(16.4)$ \\
\hline Cardiovascular disease & $21(17.2)$ \\
\hline Diabetes mellitus & $14(11.5)$ \\
\hline Active smokers & $7(5.6)$ \\
\hline Treatment with ACE/ARB, $\mathrm{n}(\%)$ & $34(27.9)$ \\
\hline \multicolumn{2}{|l|}{ Rheumatic diseases, n (\%) } \\
\hline RA & $41(33.6)$ \\
\hline SpA & $24(19.7)$ \\
\hline SLE & $13(10.7)$ \\
\hline PsA & $13(10.7)$ \\
\hline Miscellaneous* & $31(25.4)$ \\
\hline Duration of rheumatic disease $($ mean $\pm S D)$, years & $12.2 \pm 9.3$ \\
\hline \multicolumn{2}{|l|}{ Concomitant treatment, $\mathrm{n}(\%)$} \\
\hline Hydroxychloroquine & $26(21.3)$ \\
\hline Glucocorticoids & $48(39.3)$ \\
\hline cDMARDs & $80(65.6)$ \\
\hline Methotrexate & $54(44.3)$ \\
\hline Sulfasalazine & $19(15.6)$ \\
\hline Leflunomide & $13(10.7)$ \\
\hline Azathioprine & $2(1.6)$ \\
\hline Cyclophosphamide & $1(0.8)$ \\
\hline bDMARDs/tsDMARDs & $42(34.4)$ \\
\hline Anti-TNF & $28(23.0)$ \\
\hline Rituximab & $7(5.7)$ \\
\hline Abatacept & $3(2.5)$ \\
\hline Tocilizumab & $1(0.8)$ \\
\hline Sarilumabt & - \\
\hline Secukinumab & $0(0.0)$ \\
\hline Tofacitinib & $1(0.8)$ \\
\hline Baricitinib & $1(0.8)$ \\
\hline \multicolumn{2}{|l|}{ Symptoms, n (\%) } \\
\hline Dry, non-productive cough & $84(74.3)$ \\
\hline Fever & $74(64.3)$ \\
\hline Dyspnoea & $59(50.0)$ \\
\hline Arthromyalgia & $42(36.5)$ \\
\hline Anosmia/ageusia & $41(37.5)$ \\
\hline Nausea/vomiting & $39(33.9)$ \\
\hline Respiratory insufficiency, n (\%) & $54(52.5)$ \\
\hline Non-invasive oxygen supplementation, $\mathrm{n}(\%)$ & $50(41.0)$ \\
\hline Pneumonia, n (\%) & $67(54.9)$ \\
\hline $\begin{array}{l}\text { Time from disease onset to hospital admission, days (median, } \\
\text { IQR) }\end{array}$ & $7.2(4.1-10.5)$ \\
\hline Hospital admission, $\mathrm{n}(\%)$ & $69(56.6)$ \\
\hline ICU admission, $\mathrm{n}(\%)$ & $6(4.9)$ \\
\hline Time of hospital admission, days (median, IQR) & $8.0(5.0-15.2)$ \\
\hline \multicolumn{2}{|l|}{ COVID-19 specific treatment, $\mathrm{n}(\%)$} \\
\hline Hydroxychloroquine & $76(62.3)$ \\
\hline Azithromycin & $50(41.0)$ \\
\hline Glucocorticoids & $8(6.6)$ \\
\hline Lopinavir/ritonavir & $6(4.9)$ \\
\hline Anti-IL6 & $6(4.9)$ \\
\hline Anti-IL1 & $2(1.6)$ \\
\hline IVlg & $3(2.5)$ \\
\hline
\end{tabular}




\begin{tabular}{ll}
\hline Table 1 Continued & \\
\hline Recovered patients, $\mathrm{n}(\%)$ & $106(87.6)$ \\
\hline Exitus, $\mathrm{n}(\%)$ & $14(11.5)$ \\
\hline
\end{tabular}

*See online supplementary table S1.

tOne patient on double blind clinical trial with sarilumab versus placebo.

$A R B$, angiotensin-receptor blocker; bDMARDs, biological disease-modifying antirheumatic drugs; CDMARDs, conventional synthetic disease-modifying antirheumatic drugs;

ICU, intensive care units; IL, interleukin; IVIg, intravenous immunoglobulins; PsA, psoriatic arthritis; RA, rheumatoid arthritis; SLE, systemic lupus erythematosus; SpA, spondyloarthritis; TNF, tumour necrosis factor; tsDMARDs, targeted synthetic diseasemodifying antirheumatic drugs.

pulmonary disease (OR 5.36, 95\% CI 1.60-17.94) and prednisone dose $>5 \mathrm{mg} /$ day (OR 5.70, 95\% CI 1.63-19.92).

The recent outbreak of COVID-19 represents a source of concern for the management of patients with inflammatory rheumatic diseases. However, there are some reports that suggest that treatments typically used for rheumatic diseases might be effective against COVID-19. ${ }^{4}$ In our series, there was a high proportion of patients that needed hospital admission due to severity of infection (56.6\%) compared with other cohorts, which may be explained by the higher prevalence of comorbidity, particularly hypertension, the higher use of glucocorticoids or a potential selection bias towards more severe cases. ${ }^{56}$

\begin{tabular}{|c|c|c|c|}
\hline Variable & $\begin{array}{l}\text { Inpatients } \\
(n=69)\end{array}$ & $\begin{array}{l}\text { Outpatients } \\
(n=53)\end{array}$ & $P$ value \\
\hline \multicolumn{4}{|l|}{ Demographics } \\
\hline Female sex, n (\%) & $42(60.8)$ & $37(71.1)$ & 0.25 \\
\hline Age $($ mean $\pm S D)$ & $63.9 \pm 15.6$ & $50.5 \pm 14.1$ & $<0.01$ \\
\hline \multicolumn{4}{|l|}{ Comorbidity } \\
\hline Hypertension & $36(52.1)$ & $11(21.1)$ & 0.01 \\
\hline Obesity & $25(36.2)$ & $17(32.6)$ & 0.58 \\
\hline Chronic pulmonary disease & $17(24.6)$ & $3(5.7)$ & 0.01 \\
\hline Cardiovascular disease & $15(21.7)$ & $5(9.6)$ & 0.08 \\
\hline Diabetes mellitus & $11(15.9)$ & $3(5.7)$ & 0.09 \\
\hline Active smokers & $4(5.7)$ & $3(5.7)$ & 1.00 \\
\hline \multicolumn{4}{|l|}{ Concomitant treatment, $\mathrm{n}(\%)$} \\
\hline Hydroxychloroquine & $13(18.8)$ & $12(23.0)$ & 0.62 \\
\hline Glucocorticoids & $33(47.8)$ & $14(26.9)$ & 0.02 \\
\hline Low-dose prednisone ( $\leq 5 \mathrm{mg} /$ day) & $27(39.1)$ & $11(20.7)$ & 0.04 \\
\hline CDMARDs & $47(68.1)$ & $32(61.5)$ & 0.43 \\
\hline Methotrexate & $36(52.1)$ & $18(34.6)$ & 0.06 \\
\hline Leflunomide & $6(8.6)$ & $7(13.4)$ & 0.11 \\
\hline Sulfasalazine & $10(14.4)$ & $9(17.3)$ & 0.33 \\
\hline Azathioprine & $1(1.4)$ & - & - \\
\hline Cyclophosphamide & $1(1.4)$ & - & - \\
\hline bDMARDs/tsDMARDs & $20(28.9)$ & $22(42.3)$ & 0.12 \\
\hline Anti-TNF & $9(13.0)$ & $19(36.5)$ & 0.04 \\
\hline Rituximab & $7(10.1)$ & - & 0.01 \\
\hline Abatacept & $2(2.8)$ & $1(1.9)$ & - \\
\hline Tocilizumab & - & $1(1.9)$ & - \\
\hline Sarilumab* & - & - & - \\
\hline Secukinumab & - & - & - \\
\hline Tofacitinib & - & $1(1.9)$ & 0.36 \\
\hline Baricitinib & - & $1(1.9)$ & 0.36 \\
\hline
\end{tabular}

* One patient on double blind clinical trial with sarilumab versus placebo.

bDMARDs, biological disease-modifying antirheumatic drugs; cDMARDs, conventional disease-modifying antirheumatic drugs; TNF, tumour necrosis factor; tsDMARDs, targeted synthetic disease-modifying antirheumatic drugs.
Interestingly, methotrexate was a risk factor for hospital admission, but not for mortality, while other cDMARDs did not show differences. Notably, only one of our patients on tocilizumab was infected while all of our infected patients on rituximab needed hospital admission and one died. Additionally, patients on glucocorticoids had worse survival $(78.5 \%$ vs $34.2 \%, \mathrm{p}<0.01$; see online supplementary material). However, mortality rate in hospitalised patients (17.4\%) was lower compared with general population in our hospital $(20.7 \%)^{7}$

Our preliminary results suggest that COVID-19 does not have a major impact on mortality in patients with rheumatic disease. However, glucocorticoids seem to increase the risk of mortality, while methotrexate and rituximab may have an increased risk of hospital admission. These findings suggest differences in drug mechanism, which may influence COVID-19 course and emphasise the importance of further investigating the impact of immunosuppressive treatment.

Laura Nuño $\odot,{ }^{1}$ Marta Novella Navarro $\odot,{ }^{1}$ Gema Bonilla, ${ }^{1}$

Karen Franco-Gómez, ${ }^{1}$ Pilar Aguado, ${ }^{1}$ Diana Peiteado, ${ }^{1}$ Irene Monjo ๑ ,' Carolina Tornero $\odot,{ }^{1}$ Alejandro Villalba, ${ }^{1}$

Maria-Eugenia Miranda-Carus $\odot$, ${ }^{2}$ Eugenio De Miguel, ${ }^{1}$ Patricia Bogas, ${ }^{1}$ Ana Castilla-Plaza, ${ }^{1}$ Miguel Bernad-Pineda, ${ }^{1}$ Elena García-Lorenzo, Tamara Rodríguez-Araya, ${ }^{1}$ Alejandro Balsa ${ }^{2}$

${ }^{1}$ Rheumatology, La Paz University Hospital, Madrid, Spain

${ }^{2}$ Rheumatology, Instituto de Investigación Hospital Universitario La Paz (IDIPAZ), Madrid, Spain

Correspondence to Laura Nuño, Rheumatology, La Paz University Hospital, Madrid 28046, Spain; laura.nuno@salud.madrid.org

\section{Handling editor Josef S Smolen}

Acknowledgements Fundación Española de Reumatología and Instituto de Salud Carlos III, Ministry of Health, Spain, contributed to support the research contract of MNN

Contributors LN designed the registry and managed data collection. LN, MNN and $A B$ designed and drafted the work, with analysis and interpretation of data, revising it critically for important intellectual content. All coauthors made substantial contributions to acquisition of data. All coauthors revised and approved the version to be published.

Funding The authors have not declared a specific grant for this research from any funding agency in the public, commercial or not-for-profit sectors.

Competing interests $A B$ reports grants and personal fees from Abbvie, Pfizer, Novartis, Roche. Personal fees from Amgen, Sandoz, Lilly, UCB. Personal fees and non-financial support from BMS. Grants, personal fees and non-financial support from Nordic. Outside the submitted work. EdM reports investigation support from Abbvie, Novartis, Pfizer. Advisory boards from Abbvie, Novartis, Pfizer, MSD, BMS, UCB, Roche, Grunenthal, Janssen, Sanofi and Lilly. IM reports personal fees from Roche. Outside the submitted work.

Patient and public involvement Patients and/or the public were not involved in the design, or conduct, or reporting, or dissemination plans of this research.

\section{Patient consent for publication Not required.}

Ethics approval The study was approved by La Paz Ethics Committee (code PI-4186). Following the recommendations of Spanish Medicines Agency (Agencia Española de Medicamentos y Productos Sanitarios) regarding the outbreak of SARSCov-2 infection, informed consent in observational students is not necessary in terms of streamline the investigations. Published by Ministry of Health of Spain on 4 May 2020. Reference: MUH 11/2020.

Provenance and peer review Not commissioned; externally peer reviewed.

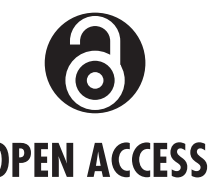

Open access This is an open access article distributed in accordance with the Creative Commons Attribution Non Commercial (CC BY-NC 4.0) license, which 
permits others to distribute, remix, adapt, build upon this work non-commercially, and license their derivative works on different terms, provided the original work is properly cited, appropriate credit is given, any changes made indicated, and the use is non-commercial. See: http://creativecommons.org/licenses/by-nc/4.0/.

(C) Author(s) (or their employer(s)) 2020. Re-use permitted under CC BY-NC. No commercial re-use. See rights and permissions. Published by BMJ.

- Additional material is published online only. To view please visit the journal online (http://dx.doi.org/10.1136/annrheumdis-2020-218054).

\section{(A) Check for updates}

To cite Nuño L, Novella Navarro M, Bonilla G, et al. Ann Rheum Dis 2020;79:1659-1661.

Received 19 May 2020

Revised 12 June 2020

Accepted 15 June 2020

Published Online First 30 June 2020

Ann Rheum Dis 2020;79:1659-1661. doi:10.1136/annrheumdis-2020-218054

\section{ORCID iDs}

Laura Nuño http://orcid.org/0000-0001-7152-0458

Marta Novella Navarro http://orcid.org/0000-0002-2200-0859

Irene Monjo http://orcid.org/0000-0002-3252-8016

Carolina Tornero http://orcid.org/0000-0001-8484-3475

Maria-Eugenia Miranda-Carus http://orcid.org/0000-0002-2334-4733

\section{REFERENCES}

1 WHO. Coronavirus disease 2019 (COVID-19) situation report - 51, 2020, 2020. Available: https://www.who.int/docs/default-source/coronaviruse/situation-reports/ 20200311- sitrep-51-covid-19.pdf?sfvrsn=1ba62e57_10 [Accessed 11 Mar 2020].

2 Yang J, Zheng Y, Gou X, et al. Prevalence of comorbidities and its effects in patients infected with SARS-CoV-2: a systematic review and meta-analysis. Int J Infect Dis 2020;94:91-5.

3 Sarzi-Puttini P, Giorgi V, Sirotti S, et al. COVID-19, cytokines and immunosuppression: what can we learn from severe acute respiratory syndrome? Clin Exp Rheumatol 2020;38:337-42.

4 Guan W-J, Ni Z-Y, Hu Y, et al. Clinical characteristics of coronavirus disease 2019 in China. N Engl J Med 2020;382:1708-20.

5 Monti S, Balduzzi S, Delvino P, et al. Clinical course of COVID-19 in a series of patients with chronic arthritis treated with immunosuppressive targeted therapies. Ann Rheum Dis 2020;79:667-8

6 Gianfrancesco MA, Hyrich KL, Gossec L, et al. Rheumatic disease and COVID-19: initial data from the COVID-19 global rheumatology alliance provider registries. Lancet Rheumatol 2020;2:e250-3.

7 Borobia AM, Carcas AJ, Arnalich F, et al. A cohort of patients with COVID-19 in a major teaching hospital in Europe. medRxiv 2020;04:20080853. 